\title{
Advanced Tokamak Investigations in Full-Tungsten ASDEX Upgrade
}

\author{
A. Bock, ${ }^{1, *}$ H. Doerk, ${ }^{1}$ R. Fischer, ${ }^{1}$ D. Rittich, ${ }^{1}$ J. Stober, ${ }^{1}$ A. Burckhart,${ }^{1}$ E. Fable, ${ }^{1}$ \\ B. Geiger, ${ }^{1}$ A. Mlynek, ${ }^{1}$ M. Reich, ${ }^{1}$ H. Zohm,${ }^{1}$ and the ASDEX Upgrade Team \\ ${ }^{1}$ Max-Planck-Institut für Plasmaphysik, Boltzmannstr. 2, 85748 Garching, Germany
}

(Dated: 2018-05-03)

\begin{abstract}
The appropriate tailoring of the $q$-profile is key to accessing Advanced Tokamak (AT) scenarios, which are of great benefit to future all-metal fusion power plants. Such scenarios depend on low collisionality $v^{*}$ which permits efficient external current drive and high amounts of intrinsic bootstrap current. At constant pressure, lowering the electron density $n_{\mathrm{e}}$ leads to a strong decrease of the collisionality with increasing electron temperature $v^{*} \sim T_{\mathrm{e}}^{-3}$. Simultaneously, the conditions for low $n_{\mathrm{e}}$ also benefit impurity accumulation. This paper reports on how radiative collapses due to central $\mathrm{W}$ accumulation were overcome by improved understanding of the changes to recycling and pumping, substantially expanded ECRH capacities for both heating and current drive, and a new solid W divertor capable of withstanding the power loads at low $n_{\mathrm{e}}$. Furthermore, it reports on various improvements to the reliability of the $q$-profile reconstruction. A candidate steady state scenario for ITER/DEMO $\left(q_{95}=5.3, \beta_{\mathrm{N}}=2.7, f_{\mathrm{bs}}>40 \%\right)$ is presented. The ion temperature profiles are steeper than predicted by TGLF, but nonlinear electromagnetic gyro-kinetic analyses with GENE including fast particle effects matched the experimental heat fluxes. A fully non-inductive scenario at higher $q_{95}=7.1$ for current drive model validation is also discussed. The results show that non-inductive operation is principally compatible with full-metal machines.
\end{abstract}

*Electronic address: alexander.bock@ipp.mpg.de 


\section{INTRODUCTION}

Future tokamak-based fusion power plants (FPPs) will feature full-metal walls and likely require long pulses or complete non-inductive operation to be economical. Scenarios which allow for such long-pulse operation are known as Advanced Tokamak (AT) scenarios. Such scenarios promise improved stability, confinement and extended pulse lengths up to fully non-inductive operation [1-3]. This is to be achieved by manipulating the toroidal current density profile $j_{\text {tor }}$ and thus the safety-factor $q$, which is a key parameter for realising these goals. Ultimately, the ideal outcome is a scenario that consistently maintains both a suitable current density profile and the corresponding $q$-profile with minimal or even no additional external current drive. Instead, large amounts of intrinsic bootstrap current density $j_{\mathrm{bs}}$ would provide the largest share of the toroidal current.

Such scenarios have been studied in many machines, including JET [4, 5], JT-60U [6], DIII-D [7, 8], and then-carbon ASDEX Upgrade (AUG) $[9,10]$.

This paper summarises the research efforts regarding AT scenarios in AUG since the conversion to a full-tungsten interior. These efforts are mainly directed towards developing credible advanced scenarios that are consistent with European proposals for a future demonstration power plant [11, 12]. Furthermore, the efforts also aim at verifying key models needed to extrapolate to future FPPs, e.g. transport models or current drive models. AUG's metal walls make it an ideal device for the development of scenarios for such future FPPs since they, too, will be faced with high-Z impurity inflow from their plasma-facing components (PFCs). Additionally, its expansive current drive and heating capacities allow for a variation of the $q$ - and kinetic profiles over a wide parameter range.

In order to access these scenarios it is necessary to at least initially tailor the $q$-profile as needed. Moreover, it is also necessary to properly diagnose it after the fact. The tailoring can be achieved through a controlled initial ramp-up of the plasma current, external current drive or intrinsic bootstrap current, the latter two of which particularly benefit from a low collisionality $v^{*}$, i.e. a low electron density $n_{\mathrm{e}}$ and/or high electron temperature $T_{\mathrm{e}}$.

In full-tungsten AUG, achieving these conditions concurrently is non-trivial. To maintain a low density, little to no additional gas puffing can be applied. Consequently, all beneficial aspects of gas puffing are absent, e.g. the influx of impurities cannot be suppressed nor can the heat flux onto the divertor be reduced.

Section II presents an overview over the efforts necessary to access AT conditions under these 
constraints. Since the essential $q$-profile reconstruction was also affected by the conversion to tungsten, section III reports on the improvements to the current profile reconstruction capabilities. With these prerequisites covered, section IV briefly summarised a proposal for a demonstration FPP non-inductive steady-state scenario before presenting the recently developed AUG advanced scenarios and comparing them with the target scenario. This includes a verification of the current understanding of plasma transport by comparing observed ion temperature profiles with modelled ones. Finally, section V gives a summary and an outlook.

\section{ACCESSING ADVANCED TOKAMAK CONDITIONS}

AT scenarios have been studied in AUG since the installation of the Motional Stark Effect (MSE) diagnostic in 1997 [13, 14]. Starting in 1999, the interior of AUG was successively covered with Tungsten. This was completed with the coverage of the strike point area and ICRF limiters in 2007 [15]. Prior and during the coverage, successful investigations of AT scenarios took place, for instance the demonstration of a fully non-inductive H-mode in 2000 [9].

With increasing $\mathrm{W}$ coverage of the PFCs, the approach to enter H-mode as well as to maintain advanced scenario conditions had to be adapted.

In order to enter $\mathrm{H}$-mode with the carbon divertor a steady gas supply before the $\mathrm{L} / \mathrm{H}$-transition was necessary. This was explained by the divertor storing the gas and releasing it during the transition. In contrast, with the tungsten divertor very little fuelling is necessary during the rampup. Conversely, the divertor no longer provides any gas during the $\mathrm{L} / \mathrm{H}$-transition. As a result, to satisfy the increased particle confinement in H-mode, significant gas puffing is necessary during the transition. This also helps to prevent ELM-free phases directly after the transition which would otherwise cause strong W-accumulation. More details on this can be found in reference [13].

Since low collisionality is a prerequisite both for efficient external current drive and high amounts of intrinsic bootstrap current, this strong gas puffing in the early discharge cannot be continued throughout the discharge for it would otherwise raise the plasma density and thus the collisionality. Alternative mitigation strategies are necessary for the bulk plasma to remain free of tungsten. A common solution still in use today is boronisation of the PFCs, which is usually done about once a month and has a noticeable effect for approximately 100 discharges [15]. Another approach to flush out impurities is by means of RF heating to increase outward particle flux in the core plasma. ICRF heating was initially used, but subsequent application of tungsten to the ICRF 
limiters turned the ICRF into a tungsten source. This can be mitigated by using boron-coated ICRF limiters as well as a novel 3-strap ICRF antenna that minimises RF image currents in the antenna frame [16].

Another means of expelling impurities with RF heating is by ECRH [17]. Before 2007, AUG's two ECRH systems were capable of delivering up to $0.8 \mathrm{MW}$ of electron heating for a total of $2 \mathrm{~s}$ each. Between 2007 and 2011 this was extended by another ECRH system capable of providing up to $4 \mathrm{MW}$ of heating for $10 \mathrm{~s}[18,19]$. Finally, the original system has now been decommissioned and is in the process of being replaced by a new system for a total of up to $8 \mathrm{MW}$ of ECRH power.

In a device like AUG that is comparably small it is not possible to simultaneously detach the divertor and maintain the necessary low central density. As a result the input power reaches the divertor plates largely without mitigation. Consequently, the divertor must be hardened to withstand continuous power deposition of over $10 \mathrm{MW}$ for several seconds. For this reason, since the 2014 campaign AUG has been equipped with a solid tungsten divertor, Div.-III. It has shown to be able to absorb heating energy of up to $100 \mathrm{MJ}$ while withstanding normalised heat fluxes $P_{\text {sep }} / R$ of up to $10 \mathrm{MW} / \mathrm{m}$ [20]. Moreover, the geometry was modified with respect to Div.-IId to allow for increased pumping efficiency which also contributes to maintaining a low $n_{\mathrm{e}}$.

Limiting the effect of tungsten on the plasma without deuterium gas puffing is a balancing act between managing the sources of impurity inflow and increasing the outward transport thereof. The primary sources whose impact can be affected are the inner and outer limiters [21, 22]. This is accomplished by an increased wall clearance of the plasma. To increase the radial transport, central EC heating is used. Indeed, a lack of central ECRH usually leads to a radiative collapse of the discharge. Insufficient wall clearance leads to an increase in the edge tungsten concentration, which can still be handled by central heating [23], albeit at possibly reduced energy confinement due to increased radiative losses. This way, even strong impurity inflows can be mitigated by strong central ECRH, although the resulting plasmas may lose their relevance as $T_{\mathrm{e}}$ increases strongly over $T_{\mathrm{i}}$.

In addition to using only a minimum of gas puffing, the bulk plasma density can be reduced further by exploiting the density pump-out phenomenon associated with the use of resonant magnetic perturbation (RMP) coils [24]. Using this, H-modes with line-averaged central densities as low as $n_{\mathrm{e}} \approx 3 \times 10^{-19} \mathrm{~m}^{-3} \approx 0.3 \times n_{\mathrm{GW}}$ at $I_{\mathrm{p}}=800 \mathrm{kA}$ have been achieved. 


\section{EQUILIBRIUM RECONSTRUCTION}

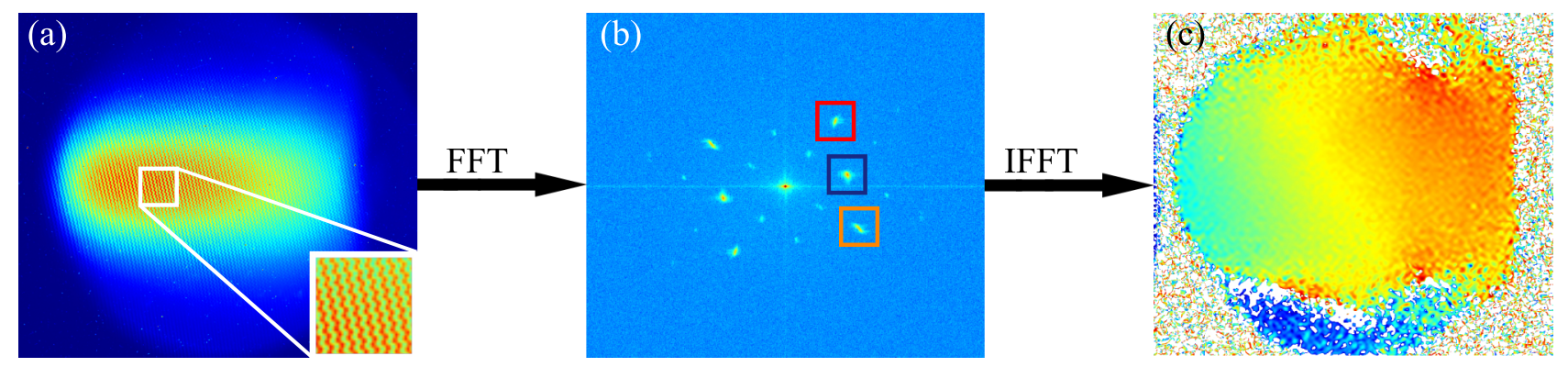

FIG. 1: Summary of the imaging MSE working principle [25]. (a) Raw image containing interference patterns caused by polarised light interacting with birefringent crystals. (b) Fourier transformed camera image with the three components of interest that include the polarisation information highlighted. (c) Backtransformed image showing the measured 2D polarisation angle.

The plasma equilibrium, in particular the $q$-profile, has profound impact not only on the plasma confinement, but also on global and local stability. The accurate determination thereof is thus a requirement for meaningful AT studies.

Since its integration the MSE diagnostic [14] that observes beam emissions from beam box 1 has been the main diagnostic for the determination of the central current density profile. During AUG's conversion from carbon to tungsten, a deterioration of the MSE diagnostic reliability was observed. As it turned out, this had two different causes that coincided: 1) a defective photoelastic modulator (PEM) caused random drifts in the measurements and 2) the $\mathrm{W}$-coverage of the PFCs strongly increased the reflectivity of the torus interior which is now able to contaminate the MSE measurements through polarised light from reflections, which has also been observed at Alcator C-Mod [26]. With the defect occurring at about the same time as the introduction of tungsten, the latter effect was masked until it was definitively confirmed in 2014 [27, 28].

As a consequence of this, the current profile reconstruction capabilities were substantially expanded in order to no longer rely on a single diagnostic. The MSE diagnostic itself is currently in the process of being upgraded to a polychromator system akin to that of C-Mod [29] that can measure and correct for the polarised background light.

In addition to this, two lines of sight of the DCN-interferometer that is used for density measurements were equipped with a polarimeter such that they can now perform Faraday rotation measurements (POL) [30]. The main advantage of this system over MSE is that measurements are 
independent of the heating scheme and therefore available under all conditions. This also provides for an automatic baseline measurement before and after the discharge. This means that as opposed to MSE systems, POL always knows its zero value. Filters are in place to prevent contamination of the measurements through reflections of polarised microwave radiation from EC heating.

Being a line integrated measurement through the plasma, POL measurements cannot offer the same localised information as MSE measurements. However, since Faraday rotation is the strongest were the magnetic field is parallel to the line of the sight, the bulk of the contribution to the signal stems from one narrow region. There, the MSE and Faraday polarimetry can be easily cross-checked.

A novel current profile diagnostic is the imaging MSE (IMSE) system that observes beam emissions from beam box 2 [25]. In contrast to the regular MSE system, the IMSE does not use PEMs to convert the polarisation of light into an intensity modulation in time. Instead, an array of optically active crystals creates multiple interference patterns that encode the polarisation information in the contrast ratios that are imaged on a camera CCD. From this, a 2D polarisation image can be generated. This is shown in figure 1. The IMSE has a broad field of view reaching well into the high-field side and due to the high light efficiency, the IMSE has a high accuracy (relative $\Delta q<0.05$ ). Additionally, the IMSE is set up to cause constructive interference between the $\pi$ - and $\sigma$-components of the beam emissions such that it can process light from both lines. This makes it independent of any broadband polarised background light. These characteristics make the IMSE diagnostic ideal for investigations of magnetohydrodynamic (MHD) core phenomena like Sawtooth crashes [25].

The expanded diagnostic coverage of the MSE, IMSE and POL systems is summarised in figure 2.

Finally, a new equilibrium code (IDE) has been developed to combine the data from all these diagnostics [31]. With equilibrium reconstruction being an ill-posed problem, most if not all equilibrium codes do not only use experimental data to yield a result but also rely on regularisation to pick one solution out of the large solution space. IDE all but entirely removes the need for regularisation by coupling the regular equilibrium reconstruction to a flux diffusion scheme. This allows to reduce the solution space based on time dependent physics rather than numerical regularisation.

In this scheme, the first time point of a discharge is calculated using experimental data and conventional regularisation. The experimental data includes all information available at AUG, i.e. magnetics, measured thermal electron and ion pressure as well as modelled fast ion pressure, 


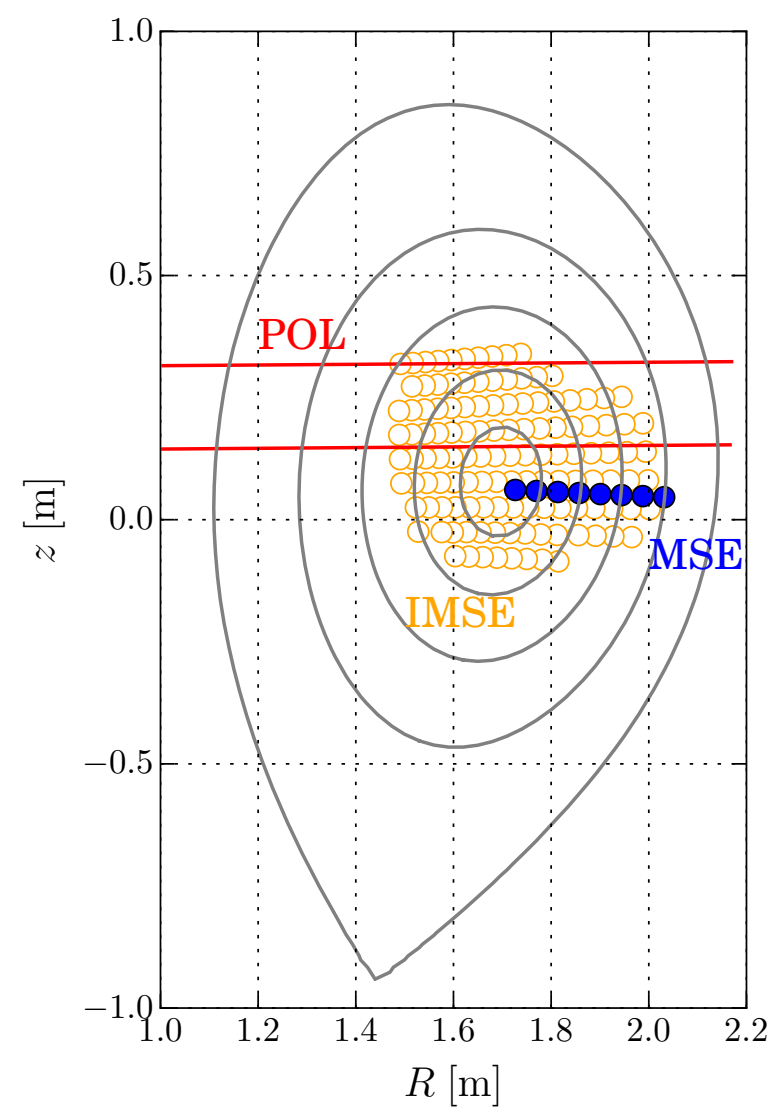

FIG. 2: Current profile diagnostic coverage in a typical H-mode discharge (\#34656). Two Faraday rotation polarimetry lines of sight shown in red, MSE lines of sight shown in blue, imaging MSE in orange. Gray contours represent flux surfaces at $\Delta \rho_{\text {pol }}=0.2$ intervals.

divertor tile currents, and optionally isoflux constraints from electron or ion temperature measurements. Subsequent time points of the reconstruction replace regularisation by the diffused toroidal flux-surface-averaged current density as a weak constraint. This diffused current is taken from the respective previous time point and is diffused according to the flux/current diffusion equation (CDE) $[32,33]$ :

$$
\sigma_{\|} \frac{\partial \psi}{\partial t}=\frac{R_{0} J^{2}}{\mu_{0} \rho_{\mathrm{tor}}} \frac{\partial}{\partial \rho_{\mathrm{tor}}}\left(\frac{G_{2}}{J} \frac{\partial \psi}{\partial \rho_{\mathrm{tor}}}\right)-\frac{V^{\prime}}{2 \pi \rho_{\mathrm{tor}}}\left(j_{\mathrm{bs}}+j_{\mathrm{CD}}\right)
$$

IDE self-consistently calculates the bootstrap current density $j_{\mathrm{bs}}$ using its own reconstructed equilibrium and measured kinetic profiles [34-36]. The externally driven current density $j_{\mathrm{CD}}$ is obtained from TORBEAM [37, 38] for ECCD and from TRANSP/NUBEAM [39-41] for neutral 


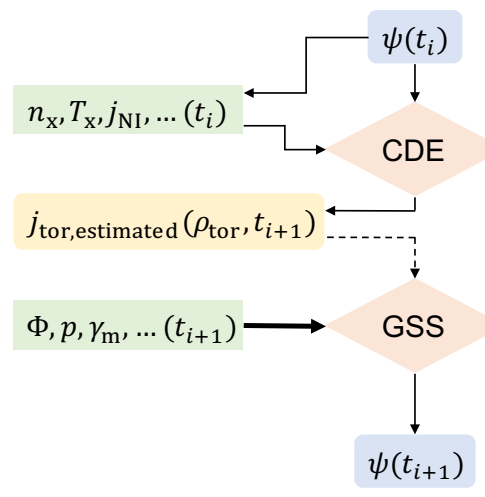

FIG. 3: Flow diagram showing IDE workflow. Initial conditions are given by the equilibrium at time point $t_{i}$, i.e. the poloidal flux matrix $\psi$ as well as the kinetic profiles $n$ or $T$ and the modelled non-inductive toroidal current contributions $j_{\mathrm{NI}}=j_{\mathrm{bs}}+j_{\mathrm{CD}}$. Applying the current diffusion equation (CDE, eq. 1) yields an estimate of $j_{\text {tor }}$ at $t_{i+1}$, which is used as a weak constraint (dashed arrow) when reconstructing the next equilibrium with a Grad-Shafranov-Solver (GSS) from all experimental data (bold arrow), e.g. magnetic information $\Phi$, pressure $p$, or MSE measurements $\gamma_{\mathrm{m}}$.

beam current drive. In the absence of internal experimental data from MSE or Faraday polarimetry, the evolution of the internal equilibrium is given by the CDE. If such data is available, then it will outweigh the $\mathrm{CDE}$ influence since the $\mathrm{CDE}$ is only included as a weak constraint. CDE information is also not included in the calculation of the result uncertainties such that they only reflect the available experimental information and are as independent of priors as possible. IDE's operation scheme is summarised in figure 3.

The diagnostics (MSE, POL, IMSE) are ultimately sensitive to the same physical quantity, namely the toroidal current enclosed in the given flux surfaces. This opens the possibility of cross-checking the data to ensure its reliability. On top of that the measurements are automatically compared to the expected evolution of the equilibrium as given by the CDE. Together the diagnostics and IDE allow for reliable equilibrium reconstruction as well as immediate identification of anomalous behaviour like flux pumping [42, 43].

\section{SCENARIO DEVELOPMENT RESULTS}

With the aforementioned necessary capabilities in place, meaningful and systematic investigations of AT scenarios are possible. In order to focus research efforts towards reactor-relevant 
scenarios, it is advantageous to define first what kind of future steady-state fusion power plant scenarios are envisaged. Many aspects play a role in constraining such scenarios and discussing them all is beyond the scope of this publication. Instead, only two shall be emphasised explicitly in the form of $0-\mathrm{D}$ arguments $[12,44]$ : for a given machine with aspect ratio $A$, there will be some maximum $\beta_{\mathrm{N}, \max }$ for a given scenario. Since fusion performance scales with $P_{\text {fus }} \sim \beta^{2}$, it suggests itself to maximise $\beta \sim \beta_{\mathrm{N}} /\left(A q_{95}\right)$ by reducing $q_{95}$. At the same time, the bootstrap current fraction $f_{\mathrm{bs}}$ scales with $\beta_{\mathrm{pol}}$, which in turn depends on $\beta_{\mathrm{N}}$ as $\beta_{\mathrm{pol}} \sim \beta_{\mathrm{N}} A q_{95}$. Evidently, to maximise both $P_{\text {fus }}$ and $f_{\text {bs }}$ would require conflicting variations of $q_{95}$. A more in-depth discussion of this discrepancy, also including 1-D modelling, can be found in reference [12]. There, a compromise scenario is proposed which is characterised by $\beta_{\mathrm{N}} \approx 3.5, q_{95} \approx 4.5$ and $H_{98}(y, 2) \approx 1.2$. At AUG, these parameters define the direction of on-going fusion power plant steady-state scenario development work.

In addition to scenarios that are potentially relevant for future fusion power plants according to these parameters, with the powerful current drive capabilities it is also possible to generate others whose primary appeal is the ability to study interesting plasma physics phenomena rather than their potential for later exploitation in a power plant.

The next subsection will report on an example of such physics studies, followed by a subsection reporting on recent scenario development efforts towards reactor-relevant conditions. Finally, transport studies under these conditions will be presented.

For all these scenarios, the starting point is given by a plasma shape with high wall clearance that helps to reduce $\mathrm{W}$ inflow from main chamber sputtering [22] without significant gas puffing [28].

An established approach to reaching a modified $q$-profile is to apply heating early in the discharge during the plasma current ramp up. As the current diffuses inward during this phase, the initial $q$-profile has a hollow shape. By early heating, the relaxation of this profile shape into a regular monotonic profile can be delayed to access various non-standard $q$-profiles even with limited current drive capabilities.

This approach has been used successfully in AUG in the past $[9,10]$. Now, having access to sufficient external current drive from both EC and NB sources, AUG does not have to make use of this approach, which is not only very sensitive to aspects like the timing of the heating but also difficult to diagnose. Instead, the plasma can be allowed to complete the regular current rampup and the modification of the $q$-profile is done in a highly controllable manner by subsequent 
external current drive.

Of course, for the purpose of studying various scenarios, manipulation of the toroidal current by means of early heating in the start-up phase remains a perfectly valid approach that is also used in AUG. The new approach that starts from an equilibrated plasma is more predictable as the evolution of the discharge after early heating in the current ramp-up phase is highly dependent on the machine conditions which can vary significantly in tungsten, especially depending on the distance from the last boronisation.

The results obtained in this manner are set apart from those of other devices in a number of ways. For instance, results from JET and JT-60U [4-6] showed the relevant conditions only transiently, whereas in AUG they are achieved stationarily, only limited by technical constraints. Another major difference to results from DIII-D [7, 8] is that the AUG results have been obtained despite harsher conditions due to impurities from the full-W walls.

It should be noted that the IMSE diagnostic was still undergoing commissioning during the studies presented in the following, and has thus not yet been used in the analysis.

\section{A. Advanced Scenarios for Basic Physics Studies}

Representative time traces of an example discharge based on an advanced scenario for basic physics studies are shown in figure 4. With an on-axis toroidal magnetic field of $2.5 \mathrm{~T}$ and a toroidal plasma current of $600 \mathrm{kA}$, the edge safety factor is $q_{95} \approx 7.1$. Following an ohmic rampup of the toroidal plasma current, the first neutral beam heating is added just after $1 \mathrm{~s}$. A total of $3 \mathrm{MW}$ of co-current off-axis ECCD is added at $1.5 \mathrm{~s}$ and maintained throughout the discharge, while a feedback-controlled increase of the total neutral beam heating power towards a target- $\beta_{\mathrm{N}}$ of $\sim 2.2$ is performed. The current drive by ECCD and NBCD is facilitated by a low line-averaged central density of $n_{\mathrm{e}} \approx 4 \times 10^{19} \mathrm{~m}^{-3}$. With this, it is possible to replace virtually all inductive toroidal current by non-inductive means. Starting at about $4.0 \mathrm{~s}$, the modelled non-inductive contributions (c.f. figure 5(e)), i.e. bootstrap current, neutral beam current and EC-driven current sum up to approximately $600 \mathrm{kA}$. The diagnosis of complete substitution of the toroidal plasma current by non-inductive contributions is further supported by a stabilisation of the loop voltage at around $0 \mathrm{~V}$ just before $4.0 \mathrm{~s}$ as shown in figure $5(\mathrm{~d})$.

Figure 5 shows profiles of $q$ and the toroidal current density, with the latter also including the modelled non-inductive contributions. Again, these non-inductive current densities add up 
to virtually the entire toroidal current density. In fact, over large parts of the minor radius, the measured, reconstructed current density in black and the modelled current densities in colour are a total match. Only in the very centre, where the plasma remains the warmest and thus retains the longest resistive time scale, is a noticeable mismatch between reconstruction and model. This points towards left-over inductive current. Furthermore, no diffusion has been taken into account for the four ECCD-peaks induced by the four used gyrotrons, which may also play a role in explaining the remaining mismatch between reconstruction and models.

Overall this makes this scenario a suitable tool for validating non-inductive current models, be it for bootstrap current or external current drivers [45]. By eliminating one contribution to the total toroidal plasma current, namely the inductive part, a major unknown is removed from the balance. It should be noted that in principle the inductive current can be calculated from the time evolution of the reconstructed equilibrium $V_{\text {loop }}=\partial \psi / \partial t$ and the conductivity of the plasma. However, the

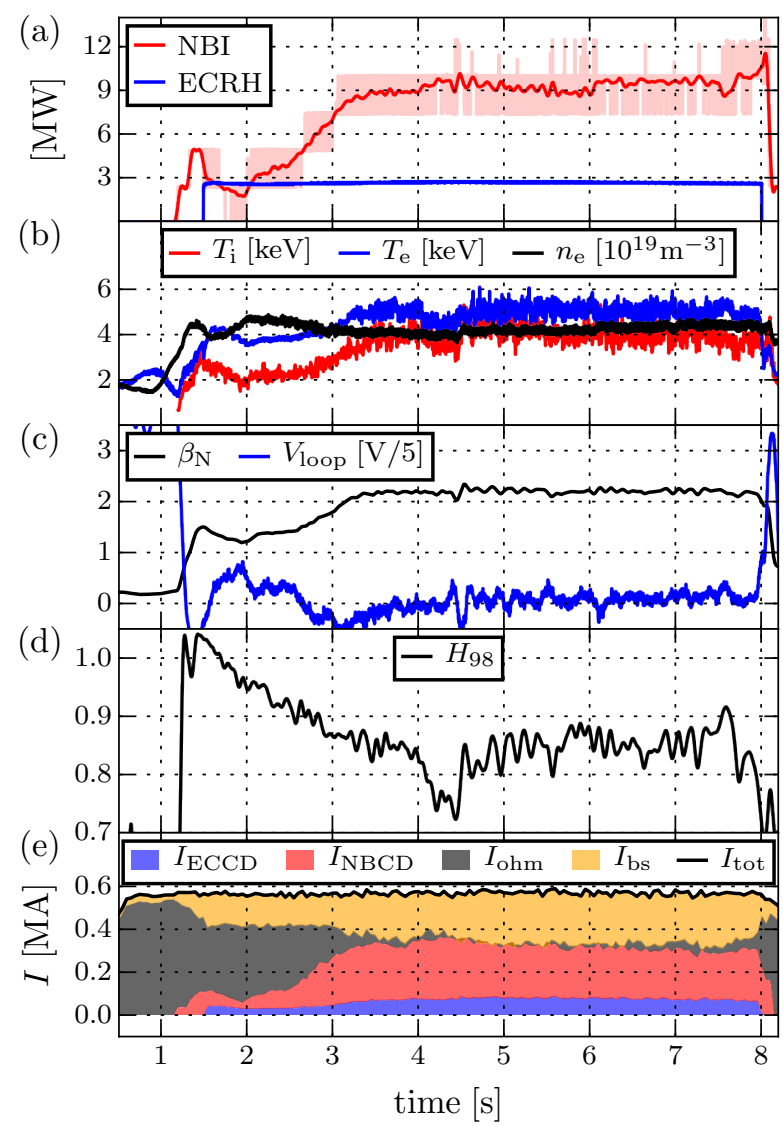

FIG. 4: (a) Heating power. (b) Core electron/ion temperatures, electron density. (c) $\beta_{\mathrm{N}}$ and loop voltage. (d) Confinement quality as given by $H_{98}(y, 2)$. (e) Composition of toroidal plasma current. (\#33379) 

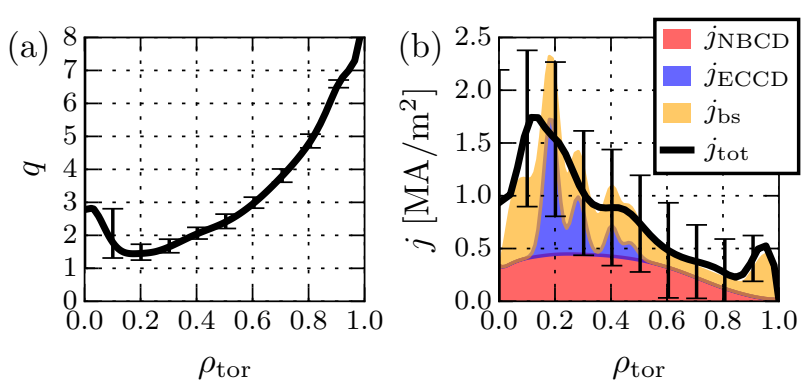

FIG. 5: (a) q-profile and (b) toroidal current density between 5-6 s in \#33379. Black curves indicate result of equilibrium reconstruction. Coloured patches in (b) representing the non-inductive contributions are calculated through modelling as described in section III with the ohmic contribution being omitted.

uncertainties on this approach are considerable and can easily reach the same order of magnitude as the toroidal current itself. Moreover, it is highly dependent on the assumptions used to calculate the equilibrium. Eliminating the inductive current in the experiment altogether sidesteps this issue and thus removes this potential point of contention. The remaining toroidal current must now be entirely described by the non-inductive models. This allows fine-tuning these models for correct application, up to the point where things like slight deviations in the sub-cm range of the external current driver geometry from the CAD models can be identified.

The uncertainties on $q$ are small since it is an integral quantity and both MSE and POL provide related integral measurements. In contrast, the uncertainties of the reconstructed toroidal current density $j_{\text {tot }}$ are larger due to it being a local quantity rather than an integral one. It is expected that the inclusion of high-resolution IMSE data will allow to significantly reduce the admittedly large uncertainties of $j_{\text {tot }}$, thereby allowing to verify the respective models to an even finer degree.

When comparing the key performance indicators of this scenario to their envisaged target values, it becomes apparent that this scenario falls short. In itself it is not suitable as a FPP scenario as formulated above, i.e. $\beta_{\mathrm{N}} \approx 2.2$ is much lower than the target value of 3.5 or above, $q_{95} \approx 7.1$ is well above the target value of 4.5 , and the confinement is lacking with $H_{98}(y, 2) \lesssim 0.9$ rather than 1.2. Still, in providing information about current drive models, this scenario can still contribute to the development of actual FPP scenarios.

In summary, although unsuitable for a future fusion power plant based on the criteria formulated above, scenarios such as this remain interesting because of their potential for validation and basic physics studies. 


\section{B. Towards Reactor-Relevant Advanced Scenarios}

Efforts to approach the aforementioned reactor-relevant advanced scenario parameters have also yielded promising results [28]. Representative time traces of such a discharge are shown in figure 6.

As in the previously presented high- $q_{95}$ example, the tailoring of the current profile again only begins after the ohmic ramp-up of the toroidal plasma current is completed. Subsequently, NBI and ECRH heating is applied. The NBI heating power is increased through a feedback control scheme towards a target- $\beta_{\mathrm{N}}$ of 2.7 , which is then maintained between $3.5 \mathrm{~s}$ and $5.0 \mathrm{~s}$.

In that interval, the discharge shows a better-than-expected confinement time with up to $H_{98}(y, 2) \approx 1.2$. Simultaneously, the loop voltage is reduced to near-zero. Moreover, modelling

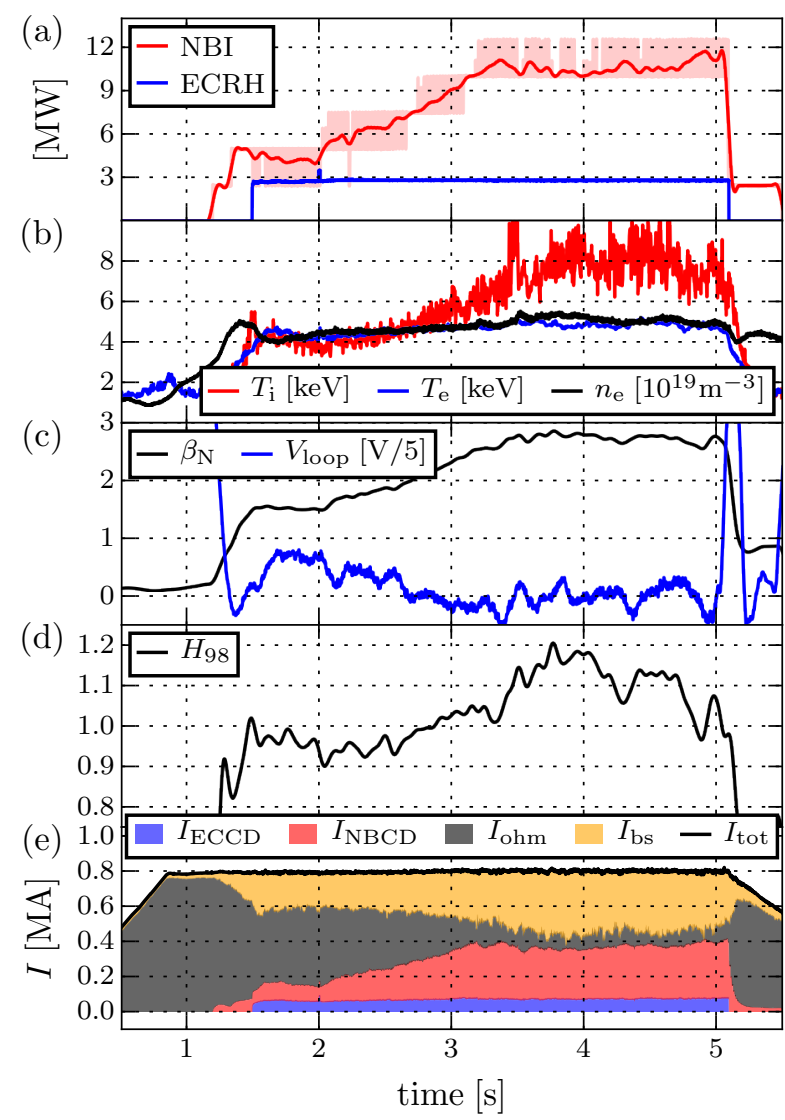

FIG. 6: (a) Heating power. (b) Core electron/ion temperatures, electron density. (c) $\beta_{\mathrm{N}}$ and loop voltage. (d) Confinement quality as given by $H_{98}(y, 2)$. (e) Composition of toroidal plasma current. Figure reproduced from [28]. (\#32305) 


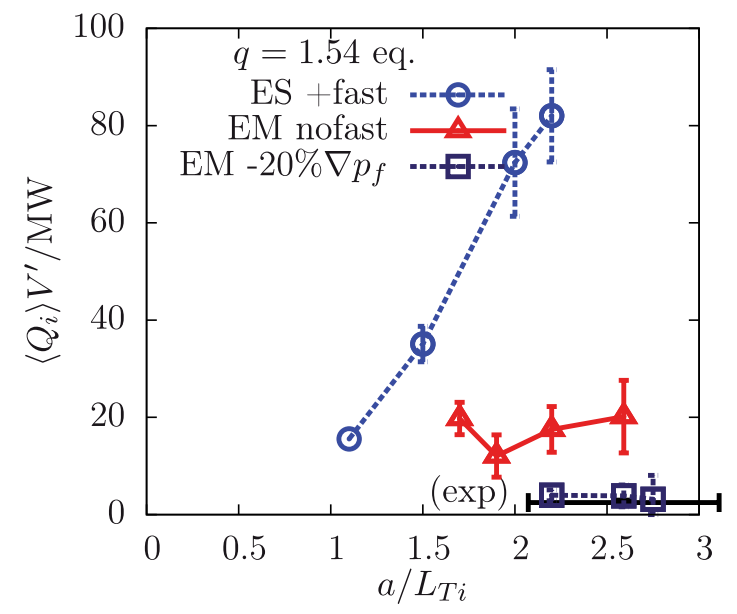

FIG. 7: Ion heat flux as a function of normalised ion temperature gradient as calculated with the GENE code at $\rho_{\text {tor }}=0.4$ in the stationary phase of \#32305. Shown are the results for slightly higher $q$ for three cases, electro-static with fast ions, electro-magnetic without fast ions, and electro-magnetic with $20 \%$ reduced fast ion pressure, which is matching the experimentally observed heat fluxes [47]. Figure reproduced from Nuclear Fusion 58, 016044 (2018). Copyright 2018 International Atomic Energy Agency.

again suggests that large parts of the toroidal plasma current are provided by non-inductive means as shown in figure 6(e). It has to be noted that the non-inductive fraction $f_{\mathrm{NI}}$ is now lower than in the high- $q_{95}$ case introduced above. A further increase in $\beta$ would help to increase $f_{\mathrm{NI}}$, but is not possible because this scenario is subject to ideal $m / n=2 / 1$ instabilities above $\beta_{\mathrm{N}} \approx 2.7[28,46]$.

With $q_{95} \approx 5.3$ already close to 4.5 and $H_{98}(y, 2) \approx 1.2$ already at the target value, the ideal $\beta$ limit now becomes the main obstacle towards reaching the desired conditions. It remains to be shown that it is possible to extend the stability space such that $\beta_{\mathrm{N}}$ can be increased from 2.7 to 3.5 , which would also be advantageous because an associated increase in the bootstrap current could also replace the remaining inductive current to reach fully non-inductive conditions.

\section{Transport Studies in Reactor-Relevant Advanced Scenarios}

The key requirement of any future fusion reactor is sufficient fusion performance. In order to be able to design future devices and scenarios to deliver this performance, understanding the physics of plasma transport and confinement is essential. Present theoretical models that are used to extrapolate to future devices must be verified by applying them to current experimental observations, which was also done with the $q_{95} \approx 5.3$ case shown above [27, 28]. 


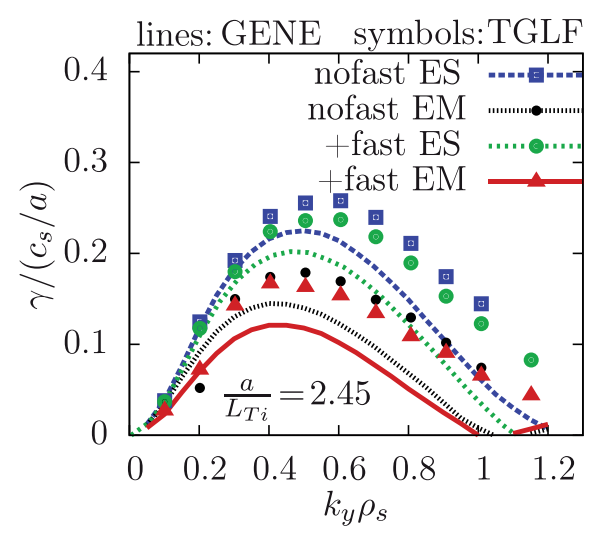

FIG. 8: Mode growth rate as calculated by the GENE code (lines) and TGLF code (symbols) in \#32305 at $\rho_{\text {tor }}=0.4$. Blue elements show electro-static results without fast ions, black ones electro-magnetic results without fast ions, while green and red represent electro-static and electro-magnetic results with fast ions, respectively [47]. Figure reproduced from Nuclear Fusion 58, 016044 (2018). Copyright 2018 International Atomic Energy Agency.

Specifically, the TGLF code $[48,49]$ was used to model heat transport in the stationary high- $\beta$ phase. It was found that TGLF over-estimates ion heat transport significantly, with normalised ion temperature gradients being under-predicted by a factor of 2-3 [28].

Motivated by results from nonlinear gyrokinetic modelling of JET discharges [50] with the GENE code [51-53], the results of TGLF were compared to the results of GENE runs [47]. One aspect of these comparisons is summarised in figure 7: when including electro-magnetic physics effects as well as fast ion effects, the ion heat flux predicted by GENE matches the experimentally observed heat flux $(\approx 2 \mathrm{MW})$ within the error bars at the observed gradient $\left(a / L_{T_{\mathrm{i}}} \approx 2.5\right)$. In contrast, at the same gradient, TGLF's predicted heat flux is more than an order of magnitude higher, i.e. that its predicted critical gradient is lower by a factor of 2-3 [47].

In order to identify the physics differences in the models of TGLF and GENE, detailed comparisons were performed. For example, in figure 8 the linear growth rates calculated by TGLF and GENE for different physics scopes are shown: electro-static and electro-magnetic, with and without fast ions. In general, it is found that for smaller $k_{y} \rho_{s}$, TGLF and GENE produce similar results. For larger $k_{y} \rho_{s}$, differences between the two codes emerge, which are more pronounced in the electro-magnetic cases, especially when fast ions are considered.

It is to be expected that the quasi-linear/non-linear results of TGLF and GENE differ when their linear results already exhibit discrepancies. In going forward, it is therefore advisable to first 
resolve these discrepancies before moving on to the later stages of the turbulent evolution.

\section{SUMMARY}

Multiple accomplishments allow for renewed advanced scenario investigations in the ASDEX Upgrade tokamak. Following the conversion to a full-tungsten machine, it is now understood how to access the low collisionality necessary for strong current profile manipulation. This is done through a combination of early gas puffing during the L-H-transition and only little additional gas puffing during the discharge, frequent boronisation to condition the plasma-facing components, in particular the ICRF limiters, and effective use of electron heating through ECRH to excite outward impurity transport. Moreover, increased wall-clearance is used to reduce the inflow of impurities from the main chamber. Optional use of RMP coils can further help to reduce the density, thereby allowing access to even lower $v^{*}$.

The current drive capabilities of AUG were successively expanded to now $6 \mathrm{MW}$ of EC heating and current drive power with $2 \mathrm{MW}$ of extra power scheduled to be available by the end of next year. Together with the two dedicated current drive sources of the NBI heating system as well as the four tangential sources (i.e. six with $2.5 \mathrm{MW}$ each), currents of the order of $>50 \%$ of the total toroidal plasma current can be driven under conditions that approach reactor-relevance.

In order to diagnose the resulting $q$-profiles, the current profile diagnostics have been improved. The conventional MSE system is being upgraded to a multi-spectral MSE system that can compensate for broadband polarised background light and is now supported by a two-chord line-integrated Faraday rotation polarimetry system and a high-resolution imaging MSE system. A newly developed equilibrium code fuses these data with other measurements while replacing non-physical regularisation with physics-based constraints by coupling a conventional Grad-Shafranov-solver to the time-dependent current diffusion equation.

With these powerful tools in place, comprehensive and well-controllable physics studies are possible. Two examples were presented in this paper. The first is a scenario at $q_{95} \approx 7.1$ that achieves virtually fully non-inductive conditions. By eliminating one unknown from the balance of currents - the inductive current - this approach allows for benchmarking of models that describe non-inductive currents, e.g. bootstrap current models or neutral beam current drive models. In contrast, the second example exists at lower $q_{95} \approx 5.3$, which approaches existing steady state DEMO scenario proposals. It offers better confinement than the higher $q_{95}$ example, but does 
so at a reduced non-inductive current fraction, and is limited to $\beta_{\mathrm{N}} \approx 2.7$ by ideal $m / n=2 / 1$ instabilities.

The improved confinement of the second example was modelled using multiple tools. It could not be reproduced with TGLF, which overestimated the ion heat transport significantly. In order to identify the cause of the discrepancy, non-linear gyrokinetic studies were performed with the GENE code. With them, it was possible to reproduce the experimental observation. Detailed comparisons between TGLF and GENE reveal deviations between the two codes that already appear in the linear phase of the transport calculations. Key players for this phenomenon include effects of high $\beta$ as well as fast ions.

The results show that non-inductive steady-state operation as planned for future FPPs with full-metal walls is achievable. Moreover, the transport investigation provides further insight into electromagnetic and fast-ion stabilisation of turbulence, which may help achieve the required confinement in future FPPs.

Future studies will focus on multiple aspects. For one thing, in order to achieve the envisaged conditions of a steady state fusion power plant $\left(\beta_{\mathrm{N}} \approx 3.5, q_{95} \approx 4.5, H_{98}(y, 2) \approx 1.2\right)$, the stability limit at $\beta_{\mathrm{N}} \approx 2.7$ must be overcome. To this end, scenarios with anomalous flux pumping [42, $54,55]$ will also be investigated. Moreover, to improve predictive capabilities, further studies will attempt to identify the physics that allows for GENE to reproduce the experimental observation such that it can be ported into codes like TGLF. Finally, the current drive capabilities will also be put to use to study various other advanced scenarios in steady state, e.g. discharges with $q_{\text {min }}>2$ that may be more resilient against the aforementioned stability limit, or discharges with extremely low density and strong shear-reversal that may strongly affect fast ion confinement.

\section{ACKNOWLEDGEMENT}

This work has been carried out within the framework of the EUROfusion Consortium and has received funding from the Euratom research and training programme 2014-2018 under grant agreement No 633053. The views and opinions expressed herein do not necessarily reflect those 
of the European Commission.

[1] M. Kikuchi, Plasma Physics and Controlled Fusion 35, B39 (1993), ISSN 07413335, URL http://stacks.iop.org/0741-3335/35/i=SB/a=003?key=crossref. $82 \mathrm{fc} 20 \mathrm{a} 1 \mathrm{fe} 0 \mathrm{fcb} 2 \mathrm{fa} 6 \mathrm{f} 004 \mathrm{a} 7 \mathrm{a} 6 \mathrm{ae} 5 \mathrm{a} 5$.

[2] R. J. Goldston, S. H. Batha, R. H. Bulmer, D. N. Hill, A. W. Hyatt, S. C. Jardin, F. M. Levinton, S. M. Kaye, C. E. Kessel, E. A. Lazarus, et al., Plasma Physics and Controlled Fusion 36 (1994), ISSN 07413335 .

[3] C. Gormezano, A. C. C. Sips, T. C. Luce, S. Ide, a. Becoulet, X. Litaudon, a. Isayama, J. Hobirk, M. R. Wade, T. Oikawa, et al., Nuclear Fusion 47, S285 (2007), ISSN 0029-5515, URL http://stacks. iop.org/0029-5515/47/i=6/a=S06?key=crossref. 62a3d2f2bad80cd96e3f1b9e93abacff.

[4] J. Hobirk, F. Imbeaux, F. Crisanti, P. Buratti, C. D. Challis, E. Joffrin, B. Alper, Y. Andrew, P. Beaumont, M. N. A. Beurskens, et al., Plasma Physics and Controlled Fusion 54, 095001 (2012), ISSN 0741-3335, URL http://stacks.iop.org/0741-3335/54/i=9/a=095001?key= crossref.af611b15eb9d34b8c1c33031215ff631.

[5] E. Joffrin, G. Gorini, C. D. Challis, N. C. Hawkes, T. C. Hender, D. F. Howell, P. Maget, P. Mantica, D. Mazon, S. E. Sharapov, et al., Plasma Physics and Controlled Fusion 44, 1739 (2002), ISSN 07413335, URL http://stacks.iop.org/0741-3335/44/i=8/a=320?key=crossref. f5b31ab8e6ff26cf147d28a299e539bf.

[6] Y. Sakamoto, G. Matsunaga, N. Oyama, T. Suzuki, N. Aiba, H. Takenaga, a. Isayama, K. Shinohara, M. Yoshida, M. Takechi, et al., Nuclear Fusion 49, 095017 (2009), ISSN 0029-5515, URL http://stacks.iop.org/0029-5515/49/i=9/a=095017?key=crossref. b566fa260f47c3b50ee508015f7d298f.

[7] F. Turco, C. C. Petty, T. C. Luce, T. N. Carlstrom, M. A. Van Zeeland, W. Heidbrink, F. Carpanese, W. Solomon, C. T. Holcomb, and J. R. Ferron, Physics of Plasmas 22, 056113 (2015), ISSN 10897674, URL http://www.scopus.com/inward/record.url?eid= 2-s2.0-84929648703\{\\&\}partnerID=tZ0tx3y1.

[8] C. Petty, J. E. Kinsey, C. Holcomb, J. DeBoo, E. J. Doyle, J. R. Ferron, A. Garofalo, A. W. Hyatt, G. Jackson, T. C. Luce, et al., Nuclear Fusion 56, 016016 (2016), ISSN 0029-5515, URL http://stacks.iop.org/0029-5515/56/i=1/a=016016?key=crossref. 
a2e7bdb3b8881f197cdc1c971b3d6214.

[9] J. Hobirk, R. C. Wolf, O. Gruber, a. Gude, S. Günter, B. Kurzan, M. Maraschek, P. J. McCarthy, H. Meister, a. G. Peeters, et al., Physical Review Letters 87, 085002 (2001), ISSN 0031-9007, URL http://link.aps.org/doi/10.1103/PhysRevLett. 87.085002.

[10] J. Stober, A. Sips, C. Angioni, C. Forest, O. Gruber, J. Hobirk, L. Horton, C. Maggi, M. Maraschek, P. Martin, et al., Nuclear Fusion 47, 728 (2007), ISSN 0029-5515, URL http: //stacks . iop.org/ 0029-5515/47/i=8/a=002?key=crossref. c6794265525a8ad1174141a22763e98f.

[11] F. Romanelli, Fusion Electricity: A roadmap to the realisation of fusion energy (2012), ISBN 978-300-040720-8.

[12] H. Zohm, F. Träuble, W. Biel, E. Fable, R. Kemp, H. Lux, M. Siccinio, and R. Wenninger, Nuclear Fusion 57, 086002 (2017), ISSN 0029-5515, URL http://iopscience.iop.org/article/ 10. 1088/1741-4326/aa739e/pdfhttp://stacks.iop.org/0029-5515/57/i=8/a=086002? key=crossref $.96 \mathrm{bea} 5938 \mathrm{e} 65688 \mathrm{df2f83a950914cbf2.}$

[13] J. Stober, A. Bock, E. Fable, R. Fischer, C. Angioni, V. Bobkov, A. Burckhart, H. Doerk, A. Herrmann, J. Hobirk, et al., Advanced Tokamak Experiments in full-W ASDEX Upgrade, $\quad$ https://nucleus.iaea.org/sites/fusionportal/Shared\%20Documents/FEC\%202016/fec2016preprints/preprint0799.pdf (2016), URL https://nucleus.iaea.org/sites/fusionportal/ SharedDocuments/FEC2016/fec2016-preprints/preprint0799.pdf.

[14] R. C. Wolf, P. J. McCarthy, F. Mast, H.-P. Zehrfeld, and ASDEX Upgrade Team, in Europhysics Conference Abstracts (Proc. of the 24th \{EPS\} Conference on Controlled Fusion and Plasma Physics, Berchtesgaden, 1997) (1997), vol. 21A, part, pp. 1509-1512.

[15] R. Neu, V. Bobkov, R. Dux, A. Kallenbach, T. Pütterich, H. Greuner, O. Gruber, A. Herrmann, C. Hopf, K. Krieger, et al., Journal of Nuclear Materials 363-365, 52 (2007), ISSN 00223115.

[16] V. Bobkov, F. Braun, R. Dux, A. Herrmann, H. Faugel, H. Fünfgelder, A. Kallenbach, R. Neu, J.-M. Noterdaeme, R. Ochoukov, et al., Nuclear Fusion 56, 084001 (2016), ISSN 0029-5515, URL http://stacks.iop.org/0029-5515/56/i=8/a=084001?key=crossref. 97938d932ea404429e6d1dbbb72c9ac8.

[17] R. Neu, R. Dux, A. Geier, A. Kallenbach, R. Pugno, V. Rohde, D. Bolshukhin, J. C. Fuchs, O. Gehre, O. Gruber, et al., Plasma Physics and Controlled Fusion 44, 811 (2002), ISSN 07413335.

[18] A. Stäbler, J. Hobirk, F. Leuterer, F. Meo, and J.-M. Noterdaeme, Fusion Science and Technology 44, 730 (2003), ISSN 1536-1055, URL https://www.tandfonline.com/doi/full/10.13182/ 
FSTO3-A411.

[19] D. Wagner, G. Grünwald, F. Leuterer, A. Manini, F. Monaco, M. J. Münich, H. Schütz, J. Stober, H. Zohm, T. Franke, et al., IEEE Transactions on Plasma Science 36, 324 (2008), ISSN 00933813.

[20] A. Herrmann, H. Greuner, N. Jaksic, M. Balden, A. Kallenbach, K. Krieger, P. de Marné, V. Rohde, A. Scarabosio, and G. Schall, Nuclear Fusion 55, 063015 (2015), ISSN 0029-5515, URL http://stacks.iop.org/0029-5515/55/i=6/a=063015?key=crossref. 12 f432860ddd77044c10afcef46be5a4.

[21] R. Dux, V. Bobkov, A. Herrmann, A. Janzer, A. Kallenbach, R. Neu, M. Mayer, H. W. Müller, R. Pugno, T. Pütterich, et al., Journal of Nuclear Materials 390-391, 858 (2009), ISSN 00223115 , URL http://dx.doi .org/10.1016/j . jnucmat.2009.01.225.

[22] R. Dux, A. Janzer, and T. Pütterich, Nuclear Fusion 51, 053002 (2011), ISSN 00295515, URL http://stacks.iop.org/0029-5515/51/i=5/a=053002?key=crossref. a5730dd57fe350a6dd574ec37d0d0591.

[23] A. Kallenbach, R. Neu, R. Dux, H. U. Fahrbach, J. C. Fuchs, L. Giannone, O. Gruber, A. Herrmann, P. T. Lang, B. Lipschultz, et al., Plasma Physics and Controlled Fusion 47 (2005), ISSN 07413335.

[24] J. C. Vallet, L. Poutchy, M. S. Mohamed-Benkadda, D. Edery, E. Joffrin, P. Lecoustey, A. L. Pecquet, A. Samain, and M. Talvard, Physical Review Letters 67, 2662 (1991), ISSN 00319007.

[25] O. P. Ford, A. Burckhart, R. McDermott, T. Pütterich, and R. C. Wolf, The Review of scientific instruments 87, 11E537 (2016), ISSN 1089-7623, URL http://dx. doi .org/10 . 1063/1. 4959873.

[26] R. T. Mumgaard, S. D. Scott, R. S. Granetz, F. M. Levinton, H. Yuh, J. Ko, N. Hawkes, and C. Holcomb, pp. 1-32 (2014).

[27] A. Bock, Ph.D. thesis, Ludwig-Maximilians-Universität München (2016), URL http: //nbn-resolving.de/urn:nbn:de:bvb:19-196277http://pubman.mpdl.mpg.de/pubman/ faces/viewItemOverviewPage. jsp?itemId=escidoc: 2303955.

[28] A. Bock, E. Fable, R. Fischer, M. Reich, D. Rittich, J. Stober, M. Bernert, A. Burckhart, H. Doerk, M. Dunne, et al., Nuclear Fusion 57, 126041 (2017), ISSN 17414326, URL http://iopscience.iop.org/10.1088/1741-4326/aa8967\{\\%\}0Ahttp://iopscience. iop.org/article/10.1088/1741-4326/aa8967http://stacks.iop.org/0029-5515/57/i= $12 / \mathrm{a}=126041$ ?key=crossref. fba1abcc2425081c03a2462289f06639.

[29] R. T. Mumgaard, S. D. Scott, and M. Khoury, Review of Scientific Instruments 87, 11E527 (2016), ISSN 0034-6748, URL http://scitation.aip.org/content/aip/journal/rsi/87/11/10. 
$1063 / 1.4959793$

[30] A. Mlynek, R. Fischer, O. Ford, P. T. Lang, B. Plöckl, and M. P. S. ASDEX Upgrade Team, Max Planck Institute for Plasma Physics, in 21st Topical Conference on High Temperature Plasma Diagnostics (HTPD 2016) (University of Wisconsin, Madison, 2016), URL http://pubman .mpdl .mpg. de/pubman/item/escidoc:2316161:3.

[31] R. Fischer, A. Bock, M. Dunne, J. C. Fuchs, L. Giannone, K. Lackner, P. J. McCarthy, E. Poli, R. Preuss, M. Rampp, et al., Fusion Science and Technology 69, 526 (2016), ISSN 15361055.

[32] G. V. Pereverzev and P. N. Yushmanov, ASTRA. Automated System for Transport Analysis in a Tokamak (2002), URL http://hdl . handle.net/11858/00-001M-0000-0027-4510-D.

[33] F. Felici, O. Sauter, S. Coda, B. Duval, T. Goodman, J.-M. Moret, and J. Paley, Nuclear Fusion 51, 083052 (2011), ISSN 0029-5515.

[34] O. Sauter, C. Angioni, and Y. R. Lin-Liu, Physics of Plasmas 6, 2834 (1999), ISSN 1070664X, URL papers://f1f22675-95a4-4828-aca2-0d710de1e56f/Paper/p1703.

[35] C. Kessel, Nuclear Fusion 34, 1221 (2002), ISSN 0029-5515.

[36] R. Hager and C. S. Chang, Physics of Plasmas 23 (2016), ISSN 10897674, URL http://dx. doi . org/10.1063/1.4945615.

[37] E. Poli, A. G. Peeters, and G. V. Pereverzev, Computer Physics Communications 136, 90 (2001), ISSN 00104655, URL http://linkinghub.elsevier.com/retrieve/pii/S0010465501001461.

[38] E. Poli, A. Bock, M. Lochbrunner, O. Maj, M. Reich, A. Snicker, A. Stegmeir, F. Volpe, N. Bertelli, R. Bilato, et al., Computer Physics Communications 225, 36 (2018), ISSN 00104655, URL https: //doi.org/10.1016/j.cpc.2017.12.018.

[39] R. Hawryluk, in Physics of Plasmas Close to Thermonuclear Conditions (Elsevier, 1981), vol. 1, pp. 19-46, URL http://linkinghub.elsevier.com/retrieve/pii/B9781483283852500091.

[40] TRANSP home page, http://w3.pppl.gov/transp, URL http://w3.pppl.gov/transp.

[41] A. Pankin, D. McCune, R. Andre, G. Bateman, and A. Kritz, Computer Physics Communications 159, 157 (2004), ISSN 00104655.

[42] I. Krebs, S. C. Jardin, S. Günter, K. Lackner, M. Hoelzl, E. Strumberger, and N. Ferraro, Physics of Plasmas 24, 102511 (2017), ISSN 1070-664X, 1706.06672, URL http://arxiv.org/abs/1706. 06672http://aip.scitation.org/doi/10.1063/1.4990704.

[43] C. C. Petty, M. E. Austin, C. T. Holcomb, R. J. Jayakumar, R. J. La Haye, T. C. Luce, M. A. Makowski, P. A. Politzer, and M. R. Wade, Physical Review Letters 102, 3 (2009), ISSN 00319007. 
[44] T. C. Luce, Fusion Science and Technology 48, 1212 (2005), ISSN 15361055.

[45] D. Rittich, Phd thesis, Universität Augsburg (2018).

[46] V. Igochine, P. Piovesan, A. Bock, L. Giannone, A. Gude, O. Kudlacek, M. Maraschek, L. Marrelli, R. McDermott, M. Reich, et al., in 44th EPS conference on Plasma Physics (European Physical Society, Belfast, 2017).

[47] H. Doerk, A. Bock, A. Di Siena, E. Fable, T. Görler, F. Jenko, and J. Stober, Nuclear Fusion 58, 016044 (2018), ISSN 0029-5515, URL http://stacks.iop.org/0029-5515/58/i=1/a=016044?key= crossref. 5b3f030f8eda4f98a6c72572b28b8f12.

[48] G. M. Staebler, J. E. Kinsey, and R. E. Waltz, Physics of Plasmas 12, 1 (2005), ISSN 1070664X.

[49] G. M. Staebler, J. E. Kinsey, and R. E. Waltz, Physics of Plasmas 14, 055909 (2007), ISSN 1070664X, URL http://link . aip.org/link/PHPAEN/v14/i5/p055909/s1\{\\&\}Agg=doi.

[50] J. Citrin, F. Jenko, P. Mantica, D. Told, C. Bourdelle, J. Garcia, J. W. Haverkort, G. M. D. Hogeweij, T. Johnson, and M. J. Pueschel, Physical Review Letters 111, 155001 (2013), ISSN 0031-9007, URL http://link.aps.org/doi/10.1103/PhysRevLett.111.155001.

[51] F. Jenko, W. Dorland, M. Kotschenreuther, and B. N. Rogers, Physics of Plasmas 7, 1904 (2000), ISSN 1070664X, URL http://adsabs.harvard.edu/cgi-bin/nph-data\{ \}query?bibcode $=2000 \mathrm{PhPl} \ldots .7 .1904\}\{\backslash \&\} 1$ ink $\left\{\backslash \_\right\}$type=ABSTRACT $\{\backslash \%\} 5$ Cnpapers2 : //publication/doi/10.1063/1.874014\{\\%\}5Cnhttp://link.aip.org/link/PHPAEN/v7/ i5/p1904/s1\{\\&\}Agg=doi.

[52] T. Görler, X. Lapillonne, S. Brunner, T. Dannert, F. Jenko, F. Merz, and D. Told, Journal of Computational Physics 230, 7053 (2011), ISSN 00219991.

[53] GENE home page, http://genecode.org.

[54] J. E. Menard, R. E. Bell, D. A. Gates, S. M. Kaye, B. P. Leblanc, F. M. Levinton, S. S. Medley, S. A. Sabbagh, D. Stutman, K. Tritz, et al., Physical Review Letters 97, 6 (2006), ISSN 00319007.

[55] S. C. Jardin, N. Ferraro, and I. Krebs, Physical Review Letters 115, 1 (2015), ISSN 10797114. 